\title{
ON DUAL SETS GENERATED BY LACUNARY POLYNOMIALS
}

\author{
HANS DOBBERTIN AND VOLKER KASTEN
}

(Communicated by Kenneth R. Meyer)

\begin{abstract}
The notion of dual sets of analytic functions has been developed by Ruscheweyh. In terms of this theory a well-known convolution theorem of Szegö states that the set of all polynomials $1+a_{1} z+\cdots+a_{n} z^{n}$ nonvanishing in the unit disc $\mathbf{D}$ is the dual hull of $(1-z)^{n}$. More general for $T=\left\{m_{1}, \ldots, m_{n}\right\}$ let $\widehat{P}_{T}$ denote the set of all lacunary polynomials $1+a_{m_{1}} z^{m_{1}}+\cdots+a_{m_{n}} z^{m_{n}}$ nonvanishing in $\mathbf{D}$. In this paper we investigate whether the sets $\widehat{P}_{T}$ are generated in a similar way. Some necessary conditions are given, and the case $|T| \leq 3$ is completely solved.
\end{abstract}

\section{INTRODUCTORY REMARKS AND NOTATIONS}

First we introduce some basic notations of the theory of dual sets of analytic functions (see [5] for details). Let $\mathscr{A}_{0}$ be the set of all functions $f(z)=1+$ $a_{1} z+\cdots$ analytic in the unit disc $\mathbf{D}$. For $\mathscr{V} \subseteq \mathscr{A}_{0}$, the first dual is defined as

$$
\mathscr{V}^{*}=\left\{f \in \mathscr{A}_{0}:(f * g)(z) \neq 0 \text { in } \mathbf{D} \text { for all } g \in \mathscr{V}\right\},
$$

where $f * g$ denotes the Hadamard product. The set du $\mathscr{V}=\mathscr{V}^{* *}$ is called the second dual or dual hull of $\mathscr{V}$. For $T=\left\{m_{1}, \ldots, m_{n}\right\}$ with $0<m_{1}<\cdots<$ $m_{n}$ let $\widehat{P}_{T}$ be the set of all lacunary polynomials $1+a_{m_{1}} z^{m_{1}}+\cdots+a_{m_{n}} z^{m_{n}}$ nonvanishing in $\mathbf{D}$, and set $\widehat{P}_{n}=\widehat{P}_{\{1, \ldots, n\}}$. The sets $\widehat{P}_{T}^{*}$ are of interest for example in the study of univalence of lacunary polynomials (see [1]). By $\widehat{Q}_{T}$ we denote the set of all $p \in \widehat{P}_{T}$ with the property that all zeros of $p$ lie on $\partial \mathbf{D}$.

We say that $\mathscr{G} \subseteq \mathscr{A}_{0}$ is generating $\mathscr{V}$ if $\mathrm{du} \mathscr{G}=\mathscr{V}$. The fundamental duality theorem [5; p. 15] states $\lambda(\mathrm{du} \mathscr{G})=\lambda(\mathscr{G})$ for compact and complete $\mathscr{G} \subseteq \mathscr{A}_{0}$ and all continuous linear complex functionals $\lambda$, which are defined on the set of all functions analytic in D endowed with the topology of locally compact convergence. Therefore dual hulls can be regarded as the analogue of convex hulls in real convexity theory, where "small" generating sets play in some sense the role of extreme points.

Received by the editors July 21, 1987 and, in revised form, April 4, 1988.

1980 Mathematics Subject Classification (1985 Revision). Primary 30C10, 30C15, 30 C50.

Key words and phrases. Convolution, dual sets, lacunary polynomials, Szegö's Theorem. 
In the following, we are dealing with sets $\mathscr{V} \subseteq \mathscr{A}_{0}$ which are single-generated (i. e., $\mathscr{V}=\operatorname{du}\{f\}$ for some $\left.f \in \mathscr{A}_{0}\right)$. If $\mathscr{V}$ contains a power series $f(z)=$ $\sum_{j=0}^{\infty} a_{j} z^{j}$ with all $a_{j} \neq 0$, then $\mathscr{V}$ can be generated by a single function $g$ only in the trivial case $\mathscr{V}=\{g(x z): x \in \overline{\mathbf{D}}\}$ (see [3]). On the other hand we have $\widehat{P}_{n}=\operatorname{du}\left\{(1-z)^{n}\right\}$. In fact, this is the duality version of Szegö's convolution theorem $\widehat{P}_{n} \circledast \widehat{P}_{n} \subseteq \widehat{P}_{n}$, where " $\circledast$ " is defined as

$$
p \circledast q=p * q * \sum_{j=0}^{n}\left(\begin{array}{l}
n \\
j
\end{array}\right)^{-1} z^{j} .
$$

Contrary to the Szegö-case $T=\{1, \ldots, n\}$ it turns out that in general $\widehat{P}_{T}$ cannot be generated by a single polynomial. A necessary condition for $\widehat{P}_{T}$ to be single-generated is the symmetry of $T=\left\{m_{1}, \ldots, m_{n}\right\}$, that is $m_{i}=m_{n}-m_{n-i}$ for $i=0,1, \ldots, n$, where $m_{0}=0$ (cf. Lemma 1). We conjecture that this condition is also sufficient:

Conjecture. $\widehat{P}_{T}$ is single-generated if and only if $T$ is symmetric.

Here we shall verify our conjecture for $|T|=2$ (Theorem A) and $|T|=3$ (Theorem C). In both cases the generating polynomials (which are unique up to rotations) are given explicitly.

\section{RESULTS}

The following lemma states some necessary conditions for $\widehat{P}_{T}$ to be generated by a single polynomial $g$.

Lemma 1. Let $0=m_{0}<m_{1}<\cdots<m_{n}, T=\left\{m_{1}, \ldots, m_{n}\right\}$ and define

$$
C_{j}=\max \left\{\left|A_{j}\right|: 1+\sum_{k \in T} A_{k} z^{k} \in \widehat{P}_{T}\right\} .
$$

Suppose that $\widehat{P}_{T}=\mathrm{du}\{g\}$ for some polynomial $g(z)=1+\sum_{j \in T} a_{j} z^{j}$. Then we have:

(1) $\left|a_{j}\right|=C_{j}(j \in T)$,

(2) $\left|a_{m_{n}}\right|=1$,

(3) $g \in \widehat{Q}_{T}$,

(4) $a_{\nu} \overline{a_{m_{n}}}=\overline{a_{m_{n}-\nu}}\left(\nu=1, \ldots, m_{n}\right)$; in particular, $T$ is symmetric, i.e., $m_{i}=m_{n}-m_{n-i}(i=0,1, \ldots, n)$.

Proof. 1. Define the functional $\lambda(f)=j$ th coefficient of $f$, then by the duality theorem

$$
\lambda\left(\widehat{P}_{T}\right)=\lambda\{g(x z):|x| \leq 1\} .
$$

This proves the assertion.

2. Clearly $C_{m_{n}} \leq 1$. On the other hand, $1+z^{m_{n}} \in \widehat{P}_{T}$ and thus $C_{m_{n}} \geq 1$. Therefore $\left|a_{m_{n}}\right| \stackrel{n}{=} C_{m_{n}}=1$. 
3. This follows immediately from (2) and $g \in \widehat{P}_{T}$.

4. Consider $\tilde{g}(z)=z^{m_{n}} \overline{g(1 / \bar{z})}$. Since $g \in \widehat{Q}_{T}, \tilde{g}$ has the same zeros as $g$. Hence $\overline{a_{m_{n}}} g=\tilde{g}$.

Theorem A. Let $0<k<n$ and $T=\{k, n\} . \widehat{P}_{T}$ is generated by a single polynomial if and only if $n=2 k$, in which case $\widehat{P}_{\{k, 2 k\}}=\mathrm{du}\left\{\left(1-z^{k}\right)^{2}\right\}$.

Proof. $T$ must be symmetric by Lemma 1(4), and thus $n=2 k$ is necessary for $\widehat{P}_{T}$ to be single-generated. The converse follows from Szegö's Theorem by the substitution $z^{k} \rightarrow z$.

We now turn to the case $T=\{k, m, n\}, 0<k<m<n$. According to Lemma $1(4), n=m+k$ is a necessary condition for $\widehat{P}_{T}$ to be single-generated. Hence in the sequel we shall only consider the case $T=\{k, m, m+k\}$. For each such $T$ we shall define a polynomial $g_{T}$, which will turn out to be a generating element of $\widehat{P}_{T}$.

First let $R_{k, m}$ be the "inner radius" of $\mu(\overline{\mathbf{D}})$, where

$$
\mu(z)=\frac{m}{m-k} z^{k}+\frac{k}{m-k} z^{m},
$$

i.e., $R_{k, m}$ is the greatest value $\rho$ such that $|w| \leq \rho$ is a subset of $\mu(\overline{\mathbf{D}})$. Choose any integers $\tilde{k}, \tilde{m}$ with $\tilde{k} k+\tilde{m} m=\operatorname{gcd}(k, m)$ in order to define

$$
g_{T}(z)=1+\frac{(-1)^{\tilde{m}+1}(m+k)}{R_{k, m}(m-k)} z^{k}+\frac{(-1)^{\tilde{k}+1}(m+k)}{R_{k, m}(m-k)} z^{m}+(-1)^{\tilde{k}+\tilde{m}} z^{m+k} .
$$

Lemma 2. Suppose that $\operatorname{gcd}(k, m)=1$. Let $\mu$ be as above. Then $\mu(\mathbf{D})$ is starlike, and the boundary $\Gamma$ of $\mu(\mathbf{D})$ is made up by the subarc $\Gamma_{0}=\Gamma \cap\{w \in$ C : $\left.|\arg w| \leq \frac{\pi}{m-k}\right\}$ and its rotations which are given by the factor $e^{i 2 \pi /(m-k)}$. $\Gamma$ has $m-k$ cusps at $\arg w=(2 l+1) \frac{\pi}{m-k}$, where $|w|$ assumes its minimum value $R_{k, m}$. The maxima of $|w|$ on $\Gamma$ satisfy $\arg w=2 l \frac{\pi}{m-k}$ and $|w|=\frac{m+k}{m-k}$. Proof. Cf. [1].

Lemma 3. (i) $R_{1, m}=1$. Hence if $T=\{k, m, m+k\}$ and $k$ divides $m$ then

$$
g_{T}(z)=1-\frac{m+k}{m-k} z^{k}+\frac{m+k}{m-k} z^{m}-z^{m+k} .
$$

for the choice $\tilde{k}=1$ and $\tilde{m}=0$.

(ii) $R_{k, m}>1$ for $\operatorname{gcd}(k, m)=1, k>1$, and there is a unique solution $\gamma \in[0, \pi / m]$ of the equation

such that

$$
\frac{k}{m}=\frac{\sin \gamma}{\sin \left(\frac{\pi}{k}-\frac{m}{k} \gamma\right)}
$$

Proof. See [1].

$$
R_{k, m}=\frac{k}{m-k} \cdot \frac{\sin \left(\frac{\pi}{k}-\frac{m-k}{k} \gamma\right)}{\sin \gamma} \text {. }
$$


Next we want to prove that $g_{T} \in \widehat{Q}_{T}$. To this end we need the following lemma:

Lemma 4 (cf. Marden [4; p. 161]). All zeros of a polynomial $p(z)=\sum_{i=0}^{n} a_{i} z^{i}$ are on the unit circle if and only if $a_{i}=u \overline{a_{n-i}}(i=0,1, \ldots, n)$ for some $u \in \partial \mathbf{D}$ and all zeros of $p^{\prime}$ lie in $\overline{\mathbf{D}}$.

Lemma 5. $g_{T} \in \widehat{Q}_{T}$ for $T=\{k, m, m+k\}$.

Proof. We can assume that $\operatorname{gcd}(k, m)=1$. Applying Lemma 4 one has to verify that for

$$
\tilde{\mu}(z)=(-1)^{\tilde{m}} \frac{m}{m-k} z^{k}+(-1)^{\tilde{k}} \frac{k}{m-k} z^{m}
$$

we have $\tilde{\mu}(z) \neq R_{k, m}$ in D. In fact this follows easily from Lemma 2, since $e^{i \pi / m-k} \mu(z)=\tilde{\mu}\left(e^{i(\tilde{m}-\tilde{k}) /(m-k) \pi} z\right)$ and therefore

$$
e^{i \pi /(m-k)} \mu(\mathbf{D})=\tilde{\mu}(\mathbf{D}) \text {. }
$$

By $g_{T}^{-1}$ we denote the polynomial of type $T$ with the property

$$
g_{T} * g_{T}^{-1}=1+\sum_{j \in T} z^{j}
$$

Defining the modified convolution

$$
f \circledast g=f * g * g_{T}^{-1}
$$

we can now state our main result:

Theorem B. $\widehat{P}_{T} \circledast \widehat{P}_{T} \subseteq \widehat{P}_{T}$ for $T=\{k, m, m+k\}$.

Note that Theorem B is the analogue of Szegö's Convolution Theorem for lacunary polynomials of type $T$. In order to prove it, we need the following Lemmas 6-9.

Lemma 6. Suppose that $\operatorname{gcd}(k, m)=1$, and let $\mu(z)=\frac{m}{m-k} z^{k}+\frac{k}{m-k} z^{m}$, $H=\mathbf{C} \backslash \mu(\mathbf{D}), R=R_{k, m}$. Then $H$ has the following property:

$$
a, b \in H \quad \Longrightarrow \quad \frac{e^{i \pi /(m-k)} a b}{R} \in H .
$$

Proof. Let $\Gamma$ and $\Gamma_{0}$ be as in Lemma 2. To prove Lemma 6, it is sufficient to assume $a, b \in \Gamma$, since $J:=\mu(\mathbf{D})$ is starlike with respect to 0 . Moreover, we may assume $a, b \in \Gamma_{0}$ due to the rotational properties of $\Gamma$ and $J$.

For $a=R e^{-i \pi /(m-k)}$ and $b \in \Gamma_{0}$ we have $e^{i \pi /(m-k)} a b / R=b \in \Gamma_{0} \subseteq H$, and a similar argument holds for the case $b=R e^{i \pi /(m-k)}$ and $a \in \Gamma_{0}$, which proves the Lemma if $a$ or $b$ are cusps of $\Gamma_{0}$.

Now let $\Gamma_{0}$ be given in polar coordinates by $r=r(\varphi),-\frac{\pi}{m-k} \leq \varphi \leq \frac{\pi}{m-k}$, and assume that $a=r(\alpha) e^{i \alpha}$ and $b=r(\beta) e^{i \beta} \in \Gamma_{0}$ are no cusps.

Case (i). $\alpha<0<\beta$. Choose $\theta>0$ such that

$$
a^{\prime}=r(\alpha-\theta) e^{i(\alpha-\theta)} \in \Gamma_{0}, \quad b^{\prime}=r(\beta+\theta) e^{i(\beta+\theta)} \in \Gamma_{0},
$$


and $a^{\prime}$ or $b^{\prime}$ is a cusp. Then $\arg \left(a^{\prime} b^{\prime}\right)=\arg (a b)$ and

$$
\left|a^{\prime} b^{\prime}\right|=r(\alpha-\theta) r(\beta+\theta) \leq r(\alpha) r(\beta)=|a b|,
$$

since $r(\alpha-\theta) \leq r(\alpha)$ and $r(\beta+\theta) \leq r(\beta)$.

Now if $e^{i \pi /(m-k)} a b / R \in J$ contrary to the Lemma, it would follow that $e^{i \pi /(m-k)} a^{\prime} b^{\prime} / R \in J$, since $J$ is starlike. But this was already shown to be impossible.

Case (ii). $\alpha<\beta<0$ or $0<\alpha<\beta$. In this case a similar argument as before can be applied, because $r(\varphi)$ has the following property on the intervals $\left[-\frac{\pi}{m-k}, 0\right]$ and $\left[0, \frac{\pi}{m-k}\right]$, respectively: $r(\alpha-\theta) r(\beta+\theta) \leq r(\alpha) r(\beta)$. To prove this inequality we mention that it is equivalent to $(\log r(\varphi))^{\prime \prime} \leq 0$ on each of these intervals. The latter inequality can be verified by an elementary but rather lengthy computation, which shall be omitted.

Lemma 7. $\widehat{Q}_{T} \circledast \widehat{Q}_{T} \subseteq \widehat{Q}_{T}$ for $T=\{k, m, m+k\}$.

Proof. Again we can restrict ourselves to the case $\operatorname{gcd}(k, m)=1$. It will be shown that the assertion is a consequence of Lemma 6.

We have $p \in \widehat{Q}_{T}$ iff $p(z)=1+\bar{A} z^{k}+A u z^{m}+u z^{m+k}$ for some $u \in \partial \mathbf{D}$ and all zeros of $p^{\prime}$ lie in $\overline{\mathbf{D}}$ (see Lemma 4), or equivalently

$$
1+\frac{m}{m+k} A z^{k}+\frac{k}{m+k} \overline{A u} z^{m} \neq 0 \text { in } \mathbf{D} \text {. }
$$

Therefore we have to prove: If $1+\frac{m}{m+k} A z^{k}+\frac{k}{m+k} \overline{A u} z^{m} \neq 0$ in $\mathbf{D}$ and $1+$ $\frac{m}{m+k} B z^{k}+\frac{k}{m+k} \overline{B v} z^{m} \neq 0$ in $\mathbf{D}(u, v \in \partial \mathbf{D})$, then

$$
1+(-1)^{\tilde{m}+1} \frac{m(m-k) R}{(m+k)^{2}} A B z^{k}+(-1)^{\tilde{k}+1} \frac{k(m-k) R}{(m+k)^{2}} \overline{A B u v} z^{m} \neq 0
$$

in D. It is easy to see that this assertion is equivalent to: $1+\frac{m}{m-k} a z^{k}+$ $\frac{k}{m-k} a z^{m} \neq 0$ in $\mathbf{D}$ and $1+\frac{m}{m-k} b z^{k}+\frac{k}{m-k} b z^{m} \neq 0$ in $\mathbf{D}$ imply $1+(-1)^{\tilde{m}+1} \frac{m}{m-k} R a b z^{k}+(-1)^{\tilde{k}+1} \frac{k}{m-k} R a b z^{m} \neq 0$ in $\mathbf{D}$, which obviously means

$$
c, d \in \mathbf{C} \backslash \mu(\mathbf{D}) \Longrightarrow \frac{c d}{R} \in \mathbf{C} \backslash \tilde{\mu}(\mathbf{D})
$$

where

$$
\mu(z)=\frac{m}{m-k} z^{k}+\frac{k}{m-k} z^{m}
$$

and

$$
\tilde{\mu}(z)=\frac{(-1)^{\tilde{m}} m}{m-k} z^{k}+\frac{(-1)^{\tilde{k}} k}{m-k} z^{m} .
$$

But $\mathbf{C} \backslash \mu(\mathbf{D})=e^{-i \pi /(m-k)}(\mathbf{C} \backslash \tilde{\mu}(\mathbf{D}))$ (see proof of Lemma 5), and thus Lemma 6 completes the proof. 
Lemma 8. Suppose that $\operatorname{gcd}(k, m)=1$. Let $y_{1}, \ldots, y_{m+k}$ be the zeros of a polynomial $q \in \widehat{P}_{T}$, where $T=\{k, m, m+k\}$, and assume $\left|y_{j}\right|=1$ for at least three indices $j$. Then $\left|y_{j}\right|=1$ for $j=1, \ldots, m+k$.

Proof. Let $q(z)=1+a_{k} z^{k}+a_{m} z^{m}+a_{m+k} z^{m+k}$ and

$$
\kappa(z)=-\frac{1+a_{k} z}{a_{m}+a_{m+k} z} .
$$

Then $q(z)=0$ iff $z^{m}=\kappa\left(z^{k}\right)$. Now $y_{i}^{k}=y_{j}^{k}$ implies $y_{i}^{m}=\kappa\left(y_{i}^{k}\right)=\kappa\left(y_{j}^{k}\right)=$ $y_{j}^{m}$; hence $y_{i}=y_{j}$, since $k$ and $m$ are relatively prime. Let us suppose that $\left|y_{j}\right|=1$ for $j=1,2,3$. We will first consider the case that $y_{1}, y_{2}$, and $y_{3}$ are pairwise distinct. Then by the preceding argument $y_{1}^{k}, y_{2}^{k}$, and $y_{3}^{k}$ are also pairwise distinct. Now $\kappa(\partial \mathbf{D})=\partial \mathbf{D}$, since $\kappa$ is a Möbius map and $\kappa(z) \in \partial \mathbf{D}$ for three different points $z=y_{j} \in \partial \mathbf{D} \quad(j=1,2,3)$. Thus $\left|a_{m+k}\right|=1$. But this means $\left|y_{1} \cdots y_{m+k}\right|=1$, and because of $\left|y_{j}\right| \geq 1$ we have $\left|y_{j}\right|=1$ for all $j$.

If $y_{1} \neq y_{2}=y_{3}$ (resp. $\left.y_{1}=y_{2}=y_{3}\right)$ then consider the system of linear equations $q\left(y_{1}\right)=0, q\left(y_{2}\right)=0, q^{\prime}\left(y_{2}\right)=0$ (resp. $q\left(y_{1}\right)=0, q^{\prime}\left(y_{1}\right)=0$, $\left.q^{\prime \prime}\left(y_{1}\right)=0\right)$, where $a_{k}, a_{m}, a_{m+k}$ are regarded as unknowns, and compute $a_{m+k}=a_{m+k}\left(y_{1}, y_{2}\right)$ (resp. $a_{m+k}=a_{m+k}\left(y_{1}\right)$ ). It is then easy to see that $\left|a_{m+k}\right|=1$.

Lemma 9. Let $p$ be any polynomial, $T=\{k, m, m+k\}$ and assume $p * \widehat{Q}_{T} \subseteq$ $\widehat{P}_{T}$. Then we have $p * \widehat{P}_{T} \subseteq \widehat{P}_{T}$.

Proof. It is easy to see that we can assume $\operatorname{gcd}(k, m)=1$. Let $q \in \widehat{P}_{T}$, denote by $y_{j}(j=1, \ldots, m+k)$ the zeros of $q$ and set $x_{j}=-y_{j}^{-1}$. Clearly, $\left|x_{j}\right| \leq 1$ and denoting by $\sigma_{j}$ the elementary symmetric functions of $\mathbf{x}=\left(x_{1}, \ldots, x_{m+k}\right)$ we have

$$
q(z)=1+\sum_{j=1}^{m+k} \sigma_{j}(\mathbf{x}) z^{j}
$$

where $\sigma_{j}(\mathbf{x})=0$ for $j \notin T$.

Therefore, if we set

$$
\begin{aligned}
& E^{m+k}=\left\{\mathbf{x} \in \mathbf{C}^{m+k}:\left|x_{j}\right| \leq 1 \text { for } j=1, \ldots, m+k\right\} \\
& T^{m+k}=\left\{\mathbf{x} \in \mathbf{C}^{m+k}:\left|x_{j}\right|=1 \text { for } j=1, \ldots, m+k\right\} \\
& L^{m+k}=\left\{\mathbf{x} \in \mathbf{C}^{m+k}: \sigma_{j}(\mathbf{x})=0 \text { for } j \notin T\right\},
\end{aligned}
$$

a polynomial $q$ belongs to $\widehat{P}_{T}$ (resp. $\widehat{Q}_{T}$ ) iff there is a $\mathbf{x} \in E^{m+k} \cap L^{m+k}$ (resp. $\left.T^{m+k} \cap L^{m+k}\right)$ such that $q(z)=1+\sum_{j=1}^{m+k} \sigma_{j}(\mathbf{x}) z^{j}$.

Now let $p * \widehat{Q}_{T} \subseteq \widehat{P}_{T}$ for a polynomial $p(z)=\sum_{j=0}^{m+k} a_{j} z^{j}$ and let us define 
$r: \mathbf{C}^{m+k} \times \mathbf{C} \longrightarrow \mathbf{C}$ by

$$
r(\mathbf{x}, z)=a_{0}+\sum_{j=1}^{m+k} a_{j} \sigma_{j}(\mathbf{x}) z^{j} .
$$

Then $r(\mathbf{x}, z) \neq 0$ on $\left(T^{m+k} \cap L^{m+k}\right) \times \mathbf{D}$, since $p * \widehat{Q}_{T} \subseteq \widehat{P}_{T}$, and it remains to prove $r(\mathbf{x}, z) \neq 0$ on $\left(E^{m+k} \cap L^{m+k}\right) \times \mathbf{D}$.

Let us assume that there would be some $(\mathbf{x}, z) \in\left(E^{m+k} \cap L^{m+k}\right) \times \mathbf{D}$ with $r(\mathbf{x}, z)=0$. Applying a continuity argument we see that there exists a $(\tilde{\mathbf{x}}, \tilde{z}) \in$ $\left(E^{m+1} \cap L^{m+1}\right) \times \mathbf{D}$ such that $r(\tilde{\mathbf{x}}, \tilde{z})=0$ and

$$
|\tilde{z}|=\min \left\{|z|: \quad(\mathbf{x}, z) \in\left(E^{m+k} \cap L^{m+k}\right) \times \mathbf{D}, r(\mathbf{x}, z)=0\right\} .
$$

From $p * \widehat{Q}_{T} \subseteq \widehat{P}_{T}$ it follows that $\left|\tilde{x}_{j}\right|<1$ for at least one $j$, and furthermore $0<|\tilde{z}|<1$, since $a_{0} \neq 0$.

Now from Lemma 8 we conclude $\left|\tilde{x}_{j}\right|<1$ for at least $m+k-2$ indices $j$, so we may assume $\left|\tilde{x}_{j}\right|<1$ for $j=1, \ldots, l$ and $\left|\tilde{x}_{j}\right|=1$ for $j=l+1, \ldots, m+k$, where $l \geq m+k-2$.

Define $R\left(x_{1}, \ldots, x_{l}, z\right)=r\left(x_{1}, \ldots, x_{l}, \tilde{x}_{l+1}, \ldots, \tilde{x}_{m+k}, z\right)$ and let

$$
\begin{gathered}
\mathbf{D}^{l}=\left\{\left(x_{1}, \ldots, x_{l}\right) \in \mathbf{C}^{l}:\left|x_{j}\right|<1, j=1, \ldots, l\right\}, \\
X=\left\{\left(x_{1}, \ldots, x_{l}\right) \in \mathbf{C}^{l}: \sigma_{j}\left(x_{1}, \ldots, x_{l}, \tilde{x}_{l+1}, \ldots, \tilde{x}_{m+k}\right)=0 \text { for } j \notin T\right\} \\
\text { and } N=\left\{\left(x_{1}, \ldots, x_{l}, z\right) \in X \times \mathbf{C}: R\left(x_{1}, \ldots, x_{l}, z\right)=0\right\} .
\end{gathered}
$$

Then $N \subseteq \mathbf{C}^{l+1}$ and $X \subseteq \mathbf{C}^{l}$ are analytic sets, $\operatorname{dim}_{\mathbf{C}} X \geq l-(m+k-3) \geq 1$ at each point of $X$ and $\left(\tilde{x}_{1}, \ldots, \tilde{x}_{l}, \tilde{z}\right) \in N$. Consider

$$
\tau: N \longrightarrow \mathbf{C}, \quad \tau\left(x_{1}, \ldots, x_{l}, z\right)=1 / z \text {. }
$$

Then $\tau$ is analytic on $N \cap\left(\mathbf{D}^{l} \times \mathbf{C}\right)$ and $|\tau|$ assumes its maximum value at the point $P=\left(\tilde{x}_{1}, \ldots, \tilde{x}_{l}, \tilde{z}\right) \in N$. Therefore $\tau \equiv 1 / \tilde{z}$ on the component of $N$ through $P$, due to the maximum principle for analytic sets (cf. [2; p. 106]). As a consequence there exists a neighborhood $U$ of $\left(\tilde{x}_{1}, \ldots, \tilde{x}_{l}\right)$ such that $R\left(x_{1}, \ldots, x_{l}, \tilde{z}\right)=0$ for all $\left(x_{1}, \ldots, x_{l}\right) \in U \cap X$. It follows that

$$
Y:=\left\{\left(x_{1}, \ldots, x_{l}\right) \in X \cap \mathbf{D}^{l}: R\left(x_{1}, \ldots, x_{l}, \tilde{z}\right)=0\right\} \neq \varnothing
$$

is open and closed in $X \cap \mathbf{D}^{l}$, and thus we have $R\left(x_{1}, \ldots, x_{l}, \tilde{z}\right)=0$ on each irreducible component of $X$ through $\left(\tilde{x}_{1}, \ldots, \tilde{x}_{l}\right)$. Now any analytic set $M \subseteq$ $\mathbf{C}^{l}$ with $\operatorname{dim}_{\mathbf{C}} M \geq 1$ must intersect $\partial \mathbf{D}^{l}$, as is well known. Therefore we have $X \cap \partial \mathbf{D}^{l} \neq \varnothing$ and thus there exists a $\left(y_{1}, \ldots, y_{l}\right) \in \partial \mathbf{D}^{l}$ with $R\left(y_{1}, \ldots, y_{l}, \tilde{z}\right)=$ 0 . Setting $\mathbf{y}=\left(y_{1}, \ldots, y_{l}, \tilde{x}_{l+1}, \ldots, \tilde{x}_{m+k}\right)$ we have $(\mathbf{y}, \tilde{z}) \in\left(E^{m+k} \cap L^{m+k}\right)$ $\times \mathbf{D}, r(\mathbf{y}, \tilde{z})=0$ and $\left|y_{j}\right|<1$ for at most $l-1$ indices $j$, since $\left|\tilde{x}_{j}\right|=1$ $(j=l+1, \ldots, m+k)$ and $\left|y_{j}\right|=1$ for at least one $j \in\{1, \ldots, l\}$.

Proceeding now by induction, we see that there must be some $\mathbf{z}=\left(z_{1}, \ldots\right.$, $\left.z_{m+k}\right)$ such that $(\mathbf{z}, \tilde{z}) \in\left(E^{m+k} \cap L^{m+k}\right) \times \mathbf{D}, r(\mathbf{z}, \tilde{z})=0$ and $\left|z_{j}\right|=1$ for 
$j=m+k-2, m+k-1, m+k$. But then $\left|z_{j}\right|=1$ for all $j$ due to Lemma 8, which is impossible, since $r(\mathbf{y}, z) \neq 0$ in $\left(T^{m+k} \cap L^{m+k}\right) \times \mathbf{D}$. This contradiction completes the proof of Lemma 9.

We are now prepared to prove Theorem B. By Lemma 7 we have $\widehat{Q}_{T} \circledast \widehat{Q}_{T} \subseteq$ $\widehat{P}_{T}$ and therefore $\widehat{Q}_{T} \circledast \widehat{P}_{T} \subseteq \widehat{P}_{T}$, according to Lemma 9. Applying Lemma 9 again we get $\widehat{P}_{T} \circledast \widehat{P}_{T} \subseteq \widehat{P}_{T}$, which completes the proof of the Theorem.

The following theorem is essentially the duality version of the Convolution Theorem B.

Theorem C. Let $0<k<m<n$ and $T=\{k, m, n\}$. Then $\widehat{P}_{T}$ is singlegenerated if and only if $n=m+k$, in which case $\widehat{P}_{T}=\mathrm{du}\left\{g_{T}\right\}$.

Proof. The "only if" part is a consequence of Lemma 1(4). To prove the "if" part, let $T=\{k, m, m+k\}$. Note that $\widehat{P}_{T}=\operatorname{du}\left\{g_{T}\right\}$ if and only if $\widehat{P}_{T}^{*}=$ $\left\{g_{T}\right\}^{*}$. Now $\widehat{P}_{T}^{*} \subseteq\left\{g_{T}\right\}^{*}$, because $g_{T} \in \widehat{P}_{T}$ (Lemma 5). So it remains to verify $\widehat{P}_{T}^{*} \supseteq\left\{g_{T}\right\}^{*}$. Let $f \in\left\{g_{T}\right\}^{*}$. Then $f * g_{T} \in \widehat{P}_{T}$ and Theorem B yields $\left(f * g_{T}\right) \circledast \widehat{P}_{T} \subseteq \widehat{P}_{T}$. It follows that $f * \widehat{P}_{T} \subseteq \widehat{P}_{T}$, and thus $f \in \widehat{P}_{T}^{*}$, which completes the proof.

By the duality theorem we get at once the following application of Theorem C (cf. also [4; p. 120]):

Corollary. Let $1+a_{k} z^{k}+a_{m} z^{m}+a_{m+k} z^{m+k} \neq 0$ in $\mathbf{D}$ and $R_{k, m}$ as before. Then we have the following sharp estimate:

$$
\left|a_{k}\right|,\left|a_{m}\right| \leq \frac{m+k}{R_{k, m}(m-k)} .
$$

\section{REFERENCES}

1. H. Dobbertin, A remark on dual sets, with application to univalent trinomials, submitted.

2. R. C. Gunning and H. Rossi, Analytic functions of several complex variables, Prentice-Hall, Inc., Englewood Cliffs, New Jersey, 1965.

3. V. Kasten and St. Ruscheweyh, On dual sets of analytic functions, Math. Nachr. 123 (1985), $277-283$.

4. W. Marden, The geometry of the zeros of a polynomial in a complex variable, Amer. Math. Soc., Providence, RI, 1949.

5. St. Ruscheweyh, Convolutions in geometric function theory, Les Presses de l'Université de Montréal, Montréal, 1982.

6. G. Szegö, Bemerkungen zu einem Satz von J. H. Grace über die Wurzeln algebraischer Gleichungen, Math. Z. 13 (1922), 28 - 55.

Institut für Mathematik, Universität HanNover, Welfengarten 1, D-3000 HanNover 1 , West Germany 